\section{(1)}

CrossMark

\title{
Evaluating 17 years of latent tuberculosis infection screening in north-west England: a retrospective cohort study of reactivation
}

\author{
Dominik Zenner ${ }^{1,2,3}$, Miranda G. Loutet ${ }^{1}$, Ross Harris (10 ${ }^{1}$, Stephen Wilson ${ }^{4}$ and \\ L. Peter Ormerod ${ }^{5,6}$
}

Affiliations: ${ }^{1}$ Centre for Infectious Disease Surveillance and Control, Public Health England, London, UK. ${ }^{2}$ Institute for Global Health, University College London, London, UK. ${ }^{3}$ National Institute for Health Research Health Protection Research Unit in Respiratory Infections, Imperial College London, London, UK. ${ }^{4}$ Dept of Respiratory and General Medicine, East Lancashire Hospitals NHS Trust, Blackburn, UK. ${ }^{5}$ Institute of Inflammation, University of Manchester, Manchester, UK. 'Lancashire Postgraduate School of Medicine and Health, University of Central Lancashire, Preston, UK.

Correspondence: Dominik Zenner, Public Health England, 61 Colindale Ave, London, NW9 5EQ, UK. E-mail: dominik.zenneraphe.gov.uk

@ERSpublications

Screening follow-up showed high TB rates in LTBI-positive, treatment-naïve individuals and treatment protection http://ow.ly/IUaW30bggkH

Cite this article as: Zenner D, Loutet MG, Harris R, et al. Evaluating 17 years of latent tuberculosis infection screening in north-west England: a retrospective cohort study of reactivation. Eur Respir J 2017; 50: 1602505 [https://doi.org/10.1183/13993003.02505-2016].

ABSTRACT Approximately 72\% of tuberculosis (TB) cases in England occur among non-UK born individuals, mostly as a result of reactivation of latent TB infection (LTBI). Programmatic LTBI screening is a key intervention of the TB strategy for England. This article reviews the results of a long-standing LTBI screening initiative in England.

A retrospective cohort was created through probabilistic linkage between LTBI screening data and national TB case notifications. Screened persons were followed until they died, became a case, emigrated or until cohort-end. TB incidence rates and rate ratios (IRR) were calculated.

97 out of 1820 individuals screened for LTBI were reported to have active TB. Crude incidence rates among LTBI-positive, treatment-naïve individuals were 4.1 and 2.3 per 100 person-years in the QuantiFERON and tuberculin skin test cohorts, respectively. Among the QuantiFERON cohort, Poisson regression showed that LTBI positivity (IRR 22.6, 95\% CI 6.8-74.6) and no chemoprophylaxis increased the probability of becoming a TB case (IRR $0.17,95 \%$ CI $0.05-0.6$ ).

We found high TB rates in LTBI-positive, treatment-naïve individuals and a strong association between no treatment and becoming a TB case, demonstrating feasibility and effectiveness of LTBI screening and providing important policy lessons for LTBI screening in England and beyond.

Received: Dec 202016 | Accepted after revision: April 202017

Earn CME accreditation by answering questions about this article. You will find these at erj.ersjournals.com/journal/cme

Support statement: DZ is affiliated with the National Institute for Health Research Health Protection Research Unit (NIHR HPRU) in Respiratory Infections at Imperial College London in partnership with Public Health England (PHE). The research received funding from the NIHR HPRU in Respiratory Infections at Imperial College London in partnership with PHE. The views expressed are those of the authors and not necessarily those of the National Health Service, the NIHR, the Department of Health or PHE.

Conflict of interest: Disclosures can be found alongside this article at erj.ersjournals.com

Copyright OERS 2017. This version is distributed under the terms of the Creative Commons Attribution NonCommercial Licence 4.0. 


\section{Introduction}

Despite major advances in global tuberculosis (TB) control and a significant reduction in cases, the global burden of TB is considerable, with an estimated 10.5 million new cases reported in 2015, and 1.4 million TB-related deaths [1]. The End TB strategy [2], developed by the World Health Organization (WHO) seeks to dramatically decrease TB incidence, mortality and catastrophic costs due to TB and aims for TB elimination ( $<1$ case per million) in low-incidence countries. Achieving these targets, and particularly the drive for $\mathrm{TB}$ elimination requires optimising all tools currently at our disposal and developing new ones [3-5].

Treatment of latent TB infection (LTBI) is highly effective in preventing TB reactivation [6, 7], and LTBI treatment has a recognised role in the End TB strategy [2]. While there is national [8] and international [9] guidance on target populations, screening modes and treatment regimens, there are still large gaps in the evidence, including better estimation of the LTBI progression risk in different population groups [9].

TB incidence in England is comparatively high (10.5 per 100000), and $73 \%$ of notified cases occur among foreign-born individuals, most $(85 \%)>2$ years after entry to the UK [10]. Recognising the need to tackle LTBI systematically, the collaborative TB strategy for England recommends, supports and funds programmatic LTBI testing and treatment in migrants from high-incidence countries [11].

Systematic testing for LTBI among migrants has been performed in the local authority area of Blackburn with Darwen since 1989. The initial scheme (1989-2001) used a single tuberculin skin test (TST) with follow-up for active cases and information/advice for TST-positive patients, but no LTBI treatment in line with former national guidance [12]. LTBI progression rates in this cohort were very high [13]. Subsequently (from 2009), an interferon $-\gamma$ release assay (IGRA)-based programme was implemented and LTBI-positive patients were offered standard LTBI treatment. Matching this entire cohort to the UK enhanced TB surveillance (ETS) system enables follow-up for active TB case progression until the end of 2014.

The aim of this study is to review the evidence of this long-standing LTBI screening initiative in England to elicit lessons learned, better quantify progression and its risk factors and to shape and inform policy around LTBI screening nationally and internationally.

\section{Methods}

Study design and cohort

A retrospective cohort study was undertaken, based on data systematically collected on all persons undergoing the Blackburn TST-based LTBI screening programme between 1989 and 2001 and the IGRA-based LTBI screening and treatment programme between 2009 and 2013. Both screening programmes were aimed at recent migrants from high $\mathrm{TB}$ incidence areas, identified through new registration with primary care or through referral from the port of arrival after exclusion of active TB. Screening was limited to persons aged $<35$ years.

The cohort was established through linking the screening databases with the UK ETS system. The screening databases contained basic demographic details, including age, sex, ethnicity, country of birth, screening dates and results, as well as details of LTBI treatment and completion. Bacille Calmette-Guerin (BCG) vaccination status was only available for the TST database. A wide range of details was available for notified TB cases, including demographic information, notification dates and details of the type of disease and treatment. Death information was derived from the national TB register (ETS), which is regularly validated through Office for National Statistics (ONS) death data.

Our primary outcome was active TB disease and the secondary outcome was incident TB. Definitions are outlined in table 1.

\section{Data linkage}

We linked the TST and QuantiFERON test (QFT) screening databases to the ETS system through deterministic matching via a common identifier (National Health Service (NHS) number), where available. In addition, and to overcome missing NHS numbers in both databases, we matched records through a validated probabilistic record linkage system based on agreement or partial agreement between demographic patient information. This methodology, initially developed by Newcombe [14] has been adapted for infectious disease work [15] and validated [16].

Individuals entered the cohort at the date of their LTBI test and exited upon notification of death or becoming a TB case, their emigration from the UK or the study end date (December 31, 2014). The emigration date was imputed, using the rules outlined below. In addition, we explored the impact of the assumptions, including emigration rates, underlying the imputation using sensitivity analysis. 
TABLE 1 Definitions

\section{Active TB disease}

Incident TB cases

Prevalent TB cases LTBI
Either having evidence of a positive culture of Mycobacterium tuberculosis complex or clinical and/or radiological evidence of TB with clinician's decision to treat with a full course of anti-TB treatment Denotified cases or those with notification dates before their LTBI testing were excluded from analysis Active cases notified $>60$ days after the LTBI test date

We would expect cases detected through screening (prevalent TB cases) to be notified within this period, but tested this assumption with a sensitivity analysis varying the cut-off between 30 and 90 days

Active cases notified within 60 days of the screening date

Either a positive TST or a positive IGRA (QuantiFERON-TB) test in the absence of active TB

A positive TST required either a positive skin reaction Heaf grade 2-4 (equivalent to $6 \mathrm{~mm}$ Mantoux reaction) in the absence of prior BCG vaccination or a Heaf grade 3-4 (equivalent to $15 \mathrm{~mm}$ Mantoux reaction) for individuals with BCG vaccination

TB: tuberculosis; LTBI: latent TB infection; TST: tuberculin skin test; IGRA: interferon- $\gamma$ release assay; BCG: bacille Calmette-Guerin.

\section{Multiple imputation and sensitivity analysis}

The duration of stay determining the exit date was imputed using length-of-stay information by visa type from the UK ONS between 2005 and 2013 [17]. Visa information was not routinely collected as part of the study, but assigned based on probability according to visa type distributions for that particular country. Those assigned family settlement/reunion visas were assumed to stay until the study end date, whereas work and study visas were randomly assigned a length of stay according to a uniform distribution, with lower and upper bounds according to the assigned duration category, and an upper bound of 10 years if assigned to the $\geqslant 4$-year duration category. In addition, age-specific ONS mortality rates were applied. Death ascertainment for cases is completed through the ETS system; however, there is a very small chance of death among the rest of the cohort and a date of death was randomly assigned to each individual, which truncates follow-up time if the imputed date precedes the end of the study period or the imputed length of stay.

The imputation procedure assumes that the remaining duration of stay at the start of the study is the length of the entire UK stay, given that no information was available on how long into the duration of stay participants were at the start of the study. This could result in an overestimate of the remaining duration of UK stay to some extent, given that the distributions used are unbiased.

Completion rates for country of birth were $99.2 \%$ (1330 out of 1341) and 54.1\% (259 out of 479) for the QFT and TST cohorts, respectively. Where country of birth was missing (13.5\% of records), this was assigned based on the Onomap software (PublicProfiler, Newcastle, UK) using forename and surname. A small number of cases could not be assigned using Onomap and were randomly assigned a country of birth based on the overall distribution of observed countries.

The imputation process was repeated 10 times to produce a multiple imputation dataset, which was analysed according to RuBIN's [18] rules; this procedure conducts a separate analysis of every dataset and takes an average of the results, with confidence intervals accounting for both within- and between-imputation uncertainty.

Although the multiple imputation dataset reflects the statistical uncertainty of the estimated cohort time based on our data, this rests on the assumption that these data provide an accurate representation of the length-of-stay distribution for the participants in this study. As this cannot be verified, we undertook sensitivity analyses using the 25th and 75th percentiles of imputed cohort time for each individual and repeated the analysis based on the sets of lower and higher values. In addition, we varied the exclusion period for incident cases between 30 and 90 days.

\section{Analysis}

The screening data were cleaned and de-duplicated, and where appropriate recoded to analysisspecific exposures and outcomes. Descriptive analysis was performed with cross-tabulation and calculation of incidence rates, means and medians, as appropriate. Significance testing was performed with Chi-squared and Fisher's exact tests, as appropriate. Single and multivariable analysis was performed using Poisson regression (suitable for rare outcomes) to estimate incidence rates and incidence rate ratios (IRR).

We performed all descriptive and multivariate analyses with Stata 13 (StataCorp, College Station, TX, USA). Simple figures were drawn using Excel 2011 (Microsoft, Redmond, WA, USA). 


\section{Results}

\section{Descriptive analysis}

Screening using TST occurred between January 1989 and November 2001 and that using QFT between January 2009 and December 2013. The latest follow-up date was December 31, 2014. The total cohort time was 6372 person-years, split into 3064 person-years for the QFT cohort and 3308 person-years for the TST cohort. The median follow-up for patients in the QFT cohort was 2.24 years (interquartile range (IQR) 1.8-2.7 years) and for patients in the TST cohort was 6.3 years (IQR 5.9-6.9 years) (table 2).

A total of 1820 persons were recorded as screened; 1341 with IGRA and 479 with TST. 366 (27.3\%) IGRAs and all TSTs were positive. $\sim 66.4 \%$ (243 out of 366 ) of IGRA-positive patients initiated LTBI treatment. The most common reason for noninitiation of treatment was nonattendance or loss to follow-up ( $n=106,86.2 \%)$, eight (6.5\%) were prevalent active cases and four (3.3\%) moved out of the area. Reasons for the remainder were unknown (figure 1). The median (IQR) time from test to treatment start was 49 (28-101) days. $10(4.1 \%)$ patients did not complete chemoprophylaxis ( $<90 \%$ of treatment days); none of these developed TB. As per protocol, no treatment occurred in the TST cohort.

The majority of tests in both cohorts were performed in a young adult population and the median age at screening was 25.1 years (IQR 22.1-28.6 years, range 9.6-44 years) and in both testing cohorts there were more males $(n=1012,55.6 \%)$ than females $(n=808,44.4 \%)$.

Most of the tests in both cohorts were performed on persons from the Indian subcontinent, and among those the largest proportions were from Pakistan (55.1\%) and India (25.3\%). There were smaller numbers of persons from sub-Saharan Africa, North Africa/Middle East and East/South-east Asia (table 2). BCG vaccination and having a TB case in the family was only systematically recorded in the TST cohort. 16 individuals were recorded to have had a case of TB in the family, six of whom developed TB, and 125 (26.1\%) persons had a BCG scar.

\section{Active cases}

Matching to the ETS system identified 83 TB patients (this includes 23 cases not reported previously [13]); most of these $(n=12)$ were identified after the end of the previous study or lost to follow-up $(n=8) .16$ cases were identified locally, but not reported nationally, as testing dates (1989-1998) predated ETS reporting.

We removed two cases from the analysis, because they occurred prior to LTBI testing; the total number of active TB cases in this cohort was therefore 97. 21 of these were from the QFT cohort and 76 were from the TST cohort. Characteristics of cases are presented in table 3. In the QFT cohort 17 active cases were LTBI-positive and four were LTBI-negative. All patients in the TST cohort were test-positive and LTBI treatment naive, and the overall proportion of active cases among the TST cohort was $15.9 \%$ (76 out of 479).

$48(50.1 \%)$ of TB cases were female, and all of them young adults at the time of case reporting (median 23.6 years, range $16-34.5$ years). Most cases were of South Asian ethnicity (Indian $n=28$, Pakistani $n=45$

TABLE 2 Baseline characteristics of the cohorts

\begin{tabular}{lcccc} 
& QFT $^{-}$ & $\mathbf{Q F T}^{+}$ & TST $^{+}$ & All subjects \\
\hline Total & 975 & 366 & 479 & 1820 \\
Total follow-up years & 2259.1 & 804.8 & 3308.3 & 6372.2 \\
Average follow-up years & $2.3(1.8-2.7)$ & $2.2(1.7-2.7)$ & $6.3(5.9-6.9)$ & $2.5(1.9-3.5)$ \\
Age at screening years & $25.2(22.1-28.7)$ & $26.4(22.8-29.5)$ & $24.3(21.6-27.9)$ & $25.1(22.1-28.6)$ \\
Female & $474(48.6)$ & $146(39.8)$ & $188(39.2)$ & $808(44.4)$ \\
World area of birth & & & & \\
$\quad$ South Asia & $777(79.7)$ & $303(82.7)$ & $442(92.2)$ & $1522(83.6)$ \\
North Africa and Middle East & $39(4.0)$ & $7(1.9)$ & $19(3.9)$ & $65(3.6)$ \\
Sub-Saharan Africa & $58(5.9)$ & $31(8.4)$ & $9(1.8)$ & $98(5.4)$ \\
Eastern Europe and Russia & $53(5.4)$ & $10(2.7)$ & $0(0)$ & $63(3.5)$ \\
Western Europe & $0(0)$ & $0(0)$ & $4(0.8)$ & $4(0.2)$ \\
East and south-east Asia & $47(4.8)$ & $12(3.2)$ & $4(0.8)$ & $63(3.5)$ \\
South America & $1(0.1)$ & $1(0)$ & $0(0)$ & $2(0.1)$ \\
Unknown & $0(0)$ & $2(0.5)$ & $1(0)$ & $3(0.2)$
\end{tabular}

Data are presented as $n$, median (interquartile range) or $n$ (\%). QFT: QuantiFERON; TST: tuberculin skin test. 


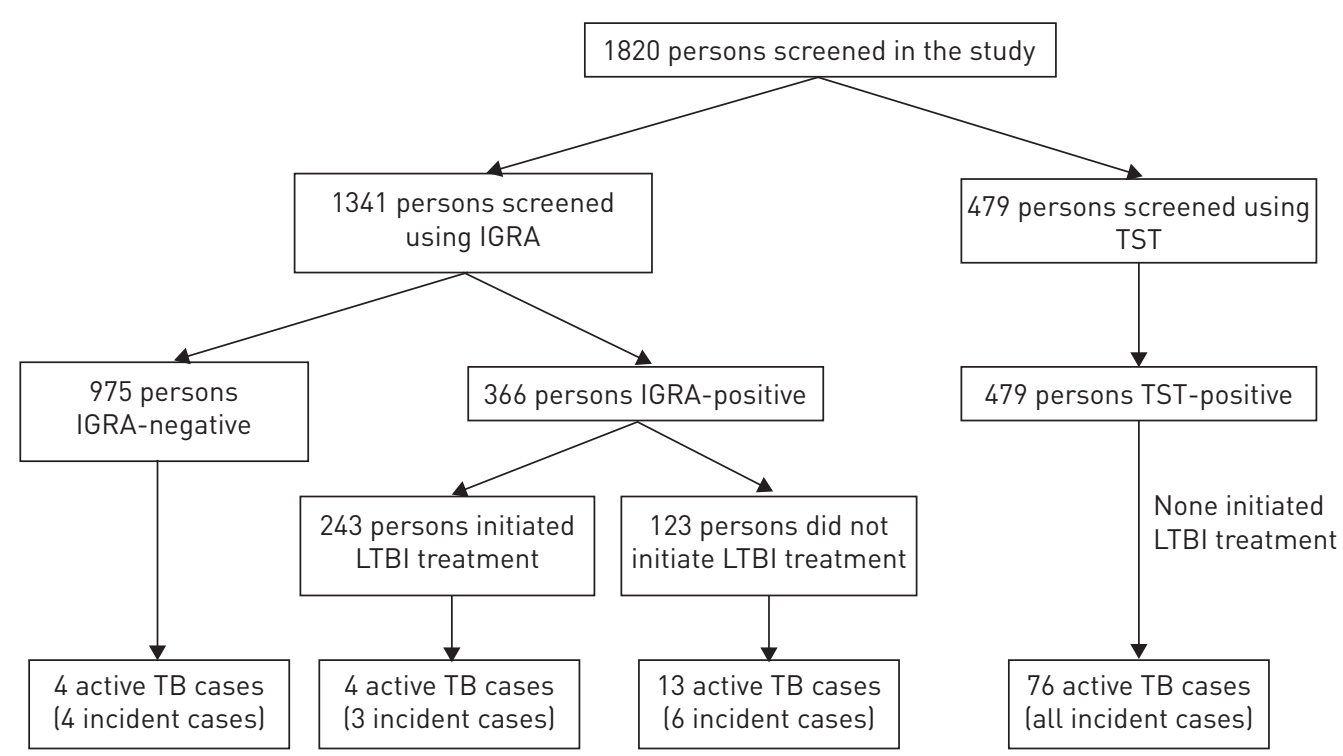

FIGURE 1 Flowchart of tuberculosis (TB) cases in the different cohorts. IGRA: interferon- $\gamma$ release assay; TST: tuberculin skin test; LTBI: latent TB infection.

and Bangladeshi $\mathrm{n}=2$ ) (table 3). Patients had entered the UK between 1989 and 2012, and approximately half (51\%) had entered the UK prior to 2000. The median (IQR) time between UK entry and the date of testing was 106 days (0-293 days).

The distribution of pulmonary and extrapulmonary TB was relatively even in the QFT cohort (extrapulmonary cases $n=9,47.4 \%$,), whereas in the TST cohort most cases had extrapulmonary TB $(n=39,65 \%)$.

\begin{tabular}{|c|c|c|c|}
\hline & QFT cohort & TST cohort & All \\
\hline Total cases & 21 & 76 & 97 \\
\hline Incident cases & 13 (61.9) & $76(100.0)$ & 89 (91.8) \\
\hline \multicolumn{4}{|l|}{ Site of disease } \\
\hline Pulmonary TB (+/- extrapulmonary TB) & $10(47.6)$ & $21(35.0)$ & 31 (38.3) \\
\hline Extrapulmonary TB only & $11(52.4)$ & $39(65.0)$ & 50 (31.0) \\
\hline Any first-line resistance & $0(0.0)$ & 1 (1.3) & $1(1.0)$ \\
\hline \multicolumn{4}{|l|}{ Time between... years } \\
\hline UK entry to case reporting & $1.3(0.9-3.0)$ & $6.7(2.7-11.6)$ & $4.4(1.9-7.9)$ \\
\hline LTBI test to case reporting & $0.2(0.1-2.0)$ & $6.8(3.6-11)$ & $4.7(1.8-8.3)$ \\
\hline Age at reporting years & $28.1(24.0-31.3)$ & $30.0(25.3-35.6)$ & $29.1(25.1-32.5)$ \\
\hline \multicolumn{4}{|l|}{ BCG } \\
\hline Yes & $4(26.7)$ & 17 (24.3) & $21(24.7)$ \\
\hline No & $11(73.3)$ & $53(75.7)$ & $64(75.3)$ \\
\hline \multicolumn{4}{|l|}{ Sex } \\
\hline Female & $9(42.9)$ & $40(52.6)$ & 49 (50.5) \\
\hline Male & $12(57.1)$ & $36(47.4)$ & 48 (49.5) \\
\hline \multicolumn{4}{|l|}{ World area of birth } \\
\hline South Asia & 19 (90.5) & $74(97.4)$ & 93 (95.9) \\
\hline Sub-Saharan Africa & 2 (9.5) & 1 (1.3) & $3(3.1)$ \\
\hline East and south-east Asia & $0(0.0)$ & $1(1.3)$ & $1(1.0)$ \\
\hline \multicolumn{4}{|l|}{ Ethnicity } \\
\hline Black African & $2(9.5)$ & $0(0.0)$ & $2(2.5)$ \\
\hline Indian & $9(42.9)$ & $21(35.6)$ & 30 (37.5) \\
\hline Pakistani & $9(42.9)$ & $36(61.0)$ & $45(56.3)$ \\
\hline Bangladeshi & $0(0.0)$ & $2(3.4)$ & $2(2.5)$ \\
\hline Mixed/other & $1(4.8)$ & $0(0.0)$ & $1(1.3)$ \\
\hline
\end{tabular}

Data are presented as $n, n(\%)$ or median (interquartile range). QFT: QuantiFERON; TST: tuberculin skin test; LTBI: latent TB infection; BCG: bacille Calmette-Guerin. 
Incident active cases

Since we analysed the effects of screening and treatment for LTBI, another important outcome of our study is incident cases, because these might have occurred as a result of LTBI reactivation. $13(61.9 \%)$ patients in the QFT cohort and all patients in the TST cohort were incident cases. 13 (10.6\%) out of the 123 untreated LTBI-positive patients in the QFT cohort developed TB, compared with four (1.7\%) of the 243 treated LTBI-positive patients in the QFT cohort ( $p=0.001)$. Excluding eight of the 21 cases in the QFT cohort as prevalent, 13 cases remained, of which four cases were LTBI-negative and nine cases were LTBI-positive. Of these positive cases, six (5.2\%) incident cases occurred among the 116 untreated patients compared with three $(1.2 \%)$ among the 242 treated patients in the QFT cohort $(\mathrm{p}=0.026)$.

For non-UK-born TB patients, time since entry to the UK is often used as proxy for time since TB infection. The median (IQR) time between UK entry and reporting date was 1.3 (0.9-3.0) years for the QFT cohort and 6.7 (2.7-11.6) years for the TST cohort (figure 2a and b). Among untreated LTBI-positive patients, early incidence is higher in the QFT group (incidence rate 236 versus 139 per 100000 person-years after 2 years); most cases $(n=54,71 \%)$ occurred $>5$ years after entry to the UK in the TST cohort.

\section{Multivariate analysis}

Incidence rates and IRRs for the QFT cohort were calculated using Poisson regression analysis. The crude TB rate in the QFT cohort was 0.6 per 100 person years, increasing to 4.1 per 100 person-years for those who were test-positive and treatment-naïve. Testing QFT-positive (IRR 22.6, 95\% CI 6.9-74.6), no treatment for LTBI (IRR 0.17, 95\% CI 0.05-0.6) and being male (IRR 0.88, 95\% CI 0.35-2.2) were associated with becoming a TB case (table $4 \mathrm{a}$ ). After adjusting for confounders, treatment for LTBI therefore reduced the risk of reactivation by an estimated $83 \%$.

In our analysis of incident cases the crude incidence rate was 0.4 per 100 person-years, increasing to 2.3 per 100 person-years among the test-positive, treatment-naïve cohort. Multivariate analysis demonstrated significance of the same exposure factors as in the overall TB case analysis, albeit with changed effect sizes (positive test IRR 12.7, 95\% CI 3.5-46.8 and chemoprophylaxis IRR 0.2, 95\% CI 0.05-0.99) (table 4).

Lastly we compared how well TST and QFT predicted TB cases among the combined test-positive, treatment-naïve cohort in multivariate analysis. QFT was a significantly better predictor of an individual becoming a TB case than TST in our cohort (IRR 2.3, 95\% CI 1.9-4.3) (table 5).

We performed a number of sensitivity analyses on our data, varying assumptions around cohort time and the definition of incident cases. While sensitivity analysis led to a change in incidence rates and effect estimates, they did not alter the main findings or the direction of effect.

\section{Discussion}

This article reports results from one of the longest running LTBI initiatives in England and perhaps globally. Cumulative reactivation rates were high in both cohorts, and particularly of note is the late
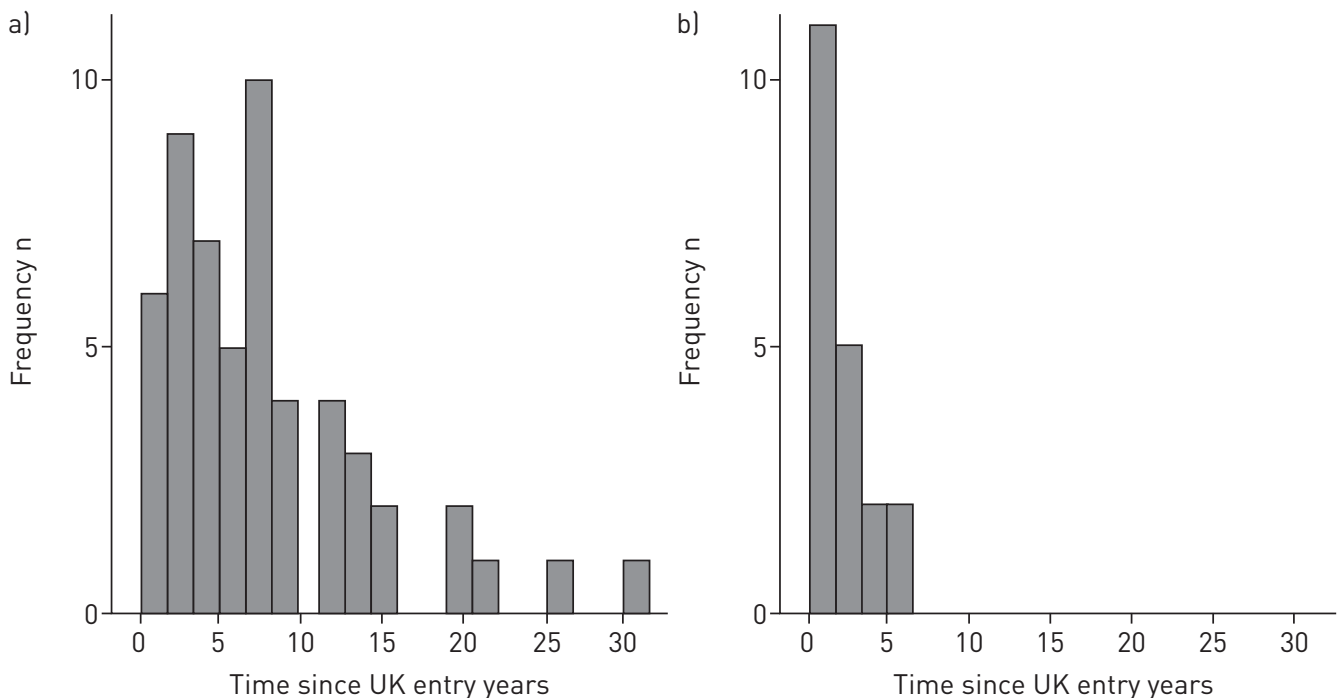

FIGURE 2 Time between UK entry and case notification for a) the tuberculin skin test cohort and b) the QuantiFERON cohort. 
TABLE 4 Incidence rates (IR) and Poisson analysis of the QuantiFERON test (QFT) cohort, with all tuberculosis (TB) cases and incident cases as outcome

All cases Incident cases

\begin{tabular}{|c|c|c|c|c|c|c|c|c|c|c|c|c|}
\hline & & \\
\hline & TB events & Person-years & IR $(95 \% \mathrm{CI})$ & IRR $(95 \% \mathrm{CI})$ & p-value & LR test & TB events & Person-years & IR $(95 \% \mathrm{CI})$ & IRR $(95 \% \mathrm{Cl})$ & $\mathrm{p}$-value & LR test \\
\hline QFT-negative & 4 & 2259.1 & $0.0018(0.0005-0.0045)$ & Ref. & & & 4 & 2259.1 & $0.0018(0.0005-0.0045)$ & Ref. & & \\
\hline QFT-positive & 15 & 804.8 & $0.0186(0.0104-0.0307)$ & $22.6435(6.8743-74.5860)$ & $<0.001$ & & 9 & 804.8 & $0.0112(0.0051-0.0212)$ & $12.7057(3.4510-46.7796)$ & $<0.001$ & 0.0012 \\
\hline No LTBI treatment ${ }^{\#}$ & 11 & 265.6 & $0.0414(0.0207-0.0741)$ & Ref. & & & 6 & 265.6 & $0.0226(0.0083-0.0492)$ & Ref. & & \\
\hline LTBI treatment ${ }^{\#}$ & 4 & 539.3 & $0.0074(0.0020-0.0190)$ & $0.1711(0.0486-0.6020)$ & 0.0060 & 0.0014 & 3 & 539.3 & $0.0056(0.0011-0.0163)$ & $0.2249(0.0508-0.9967)$ & 0.049 & 0.039 \\
\hline Female & 8 & 1371.7 & $0.0058(0.0025-0.0115)$ & Ref. & & & 5 & 1371.7 & $0.0036(0.0012-0.0085)$ & Ref. & & \\
\hline Male & 11 & 1690.1 & $0.0065(0.0032-0.0116)$ & $0.8780(0.3546-2.1739)$ & 0.7790 & 0.0200 & 8 & 1690.1 & $0.0047(0.0020-0.0093)$ & $1.1095(0.3621-3.3999)$ & 0.856 & 0.8 \\
\hline Age & & & & $-1.0239(0.9390-1.1165)$ & 0.5930 & 0.1800 & & & & $1.0076(0.9062-1.1205)$ & 0.888 & 0.9 \\
\hline \multicolumn{13}{|l|}{ Area of birth } \\
\hline Indian subcontinent & 17 & 2432.3 & $0.0070(0.0041-0.0112)$ & Ref. & & & 12 & 2432.3 & $0.0049(0.0025-0.0086)$ & Ref. & & \\
\hline Sub-Saharan Africa & 2 & 248.8 & $0.0080(0.0010-0.0290)$ & $0.7514(0.1491-3.7856)$ & 0.7290 & & 1 & 248.8 & $0.0040(0.0001-0.0224)$ & $0.5293(0.0657-4.2641)$ & 0.55 & \\
\hline Rest of world & 0 & 382.9 & $0.0000(0.0000-0.0096)$ & $0.0000(0.0000-0.0000)$ & $<0.001$ & 0.1140 & 0 & 382.9 & $0.0000(0.0000-0.0096)$ & $0.0000(0.0000-0.0000)$ & $<0.001$ & 0.2 \\
\hline
\end{tabular}


TABLE 5 Incidence rates (IR) and Poisson analysis of predictive value of tuberculin skin test (TST) versus QuantiFERON test (QFT) in test-positive, treatment-naïve cohort; outcome is tuberculosis (TB) case

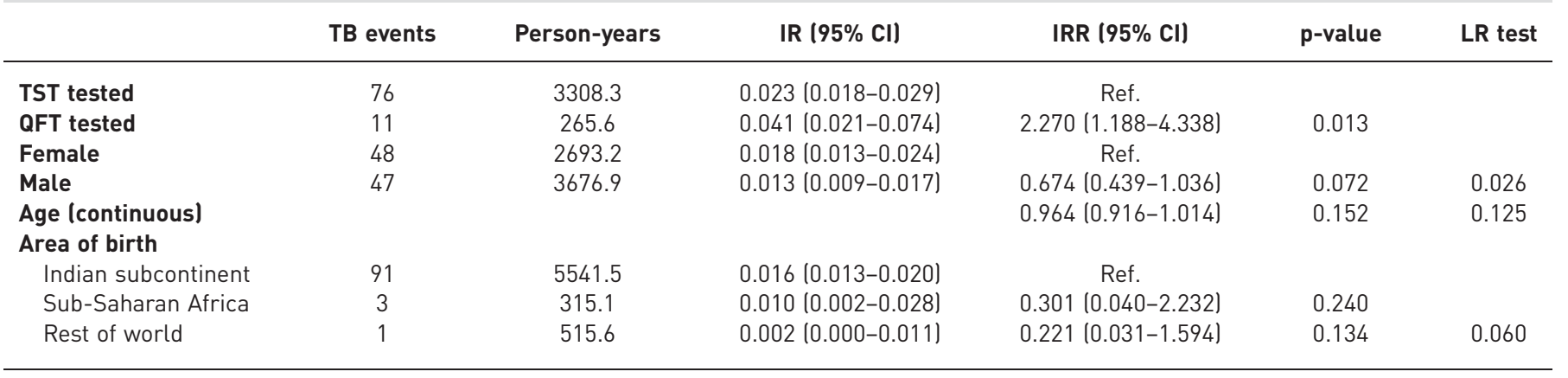

Data are presented as $n$, unless otherwise stated. IRR: incidence rate ratio; LR: likelihood ratio.

reactivation rate in the TST cohort. Follow-up time in the QFT cohort was shorter, and during the observation time, QFT positivity appears to be better correlated with becoming a TB case than TST positivity. We confirmed the protective effect of LTBI treatment. Our study did not explore important secondary effects of such a screening programme, such as awareness raising or facilitating access to care, but these should be mentioned as other important effects of such a programme to support TB control.

We present an update on previously published results in the TST cohort, confirming an even greater reactivation rate than previously thought [13]. In keeping with previous research, we confirmed the better predictive value of QFT compared with TST in our cohort [19]; this is in contrast to current recommendations from the National Institute for Health and Care Excellence [8]. The comparative performance of these tests is particularly important when deciding on the best test strategy in the context of programmatic LTBI screening.

LTBI reactivation rates have been the subject of intense debate and estimates of progression vary widely. For example, RANGAKA et al. [20] estimated progression rates of between four and 48 cases per 1000 person-years in IGRA-positive individuals with a median follow-up time of 4 years. Another meta-analysis estimated progression rates to be $2.7 \%$ and $1.5 \%$ for IGRA and TST, respectively, rising to $6.8 \%$ and $2.4 \%$, respectively if limiting to high-risk groups [19]. The same authors performed another systematic review with much higher reactivation estimates (8-15\%) for IGRA-positive individuals, mostly in contact-tracing settings [21]. Conversely, a pan-European study estimated a reactivation rate of nine and 12 per 1000 person-years for QFT and T-Spot IGRA tests, respectively [22].

Some of this variation can be explained by individual or environmental risk factors, such as proximity of recent contact with a pulmonary case or immunosuppression. There is consensus on the need to define screening target groups well [9], and more detailed description of progression risk factors has been performed by KRUISHAAR et al. [23], although it may be difficult to implement highly specific screening target groups for practical or societal reasons. Within these limits, the art of defining the most appropriate target group must carefully weigh reactivation risk, costs and benefits [24], as has been done in the national migrant LTBI testing and treatment programme in England.

Some of our findings are expected and have previously been described in the literature, such as the superiority of IGRA to predict LTBI reactivation [19-21], although both tests are recommended by the WHO [9], or the protective effect of chemotherapy to prevent TB cases [6, 7]. However, the high late TB rate in the TST cohort was unexpected, and instead of an initial peak, case numbers remained stable and high over a number of years. While the possibility of re-infection (e.g. through travel to high-incidence countries [25]) cannot be dismissed, both data and local knowledge appears to point to a relatively stable population and therefore potentially genuine observation of late reactivations. While acknowledging the limitations of TST (e.g. using Heaf test) and potential differences comparing the cohorts, these are interesting findings. Therefore, further work is needed to confirm these findings and elicit potential causes.

Our study has a number of strengths and limitations. We report observational data from a well-recorded, sizable cohort with long follow-up times, although observational data have well-known limitations. The length of the follow-up time is relevant, as due to the scarcity of observed reactivation data most knowledge is based on mathematical modelling only. In addition, the study benefits from its data-linkage to ETS with near complete case ascertainment in the UK. The probabilistic matching used has been validated before, and all detected cases have been scrutinised, so that the probability of over-ascertainment is very low, although there is a small probability that cases have been missed and under-ascertained. 
However, a key limitation is that for most individuals, the date of exit out of the UK was unknown. We minimised this limitation through using multiple imputation based on known personal characteristics, and by using sensitivity analysis to model an upper and lower estimate of UK stay. Apart from the uncertainty of length of stay, the absence of observed exit data could have led to an under-ascertainment of cases occurring outside of the UK. While we are confident that we minimised the impact of these missing data, the most likely effect of the combined limitations noted above would be an underestimation of TB reactivation rates. Alternatively, if we underestimated length of stay for temporary visa types, this may lead to over-ascertainment of late cases and could be an alternative explanation for the rate of TB not decreasing as expected. Another limitation is the absence of information on comorbidities, which were thus not adjusted for in our models. Given that this was a young community cohort, and given that none of the TB cases had recorded immunosuppression, it is unlikely that this would have changed results. Lastly, it is also worth noting that the reading threshold influences positivity and effect size for the TST cohort. National (UK) recommendations were followed to determine positivity, but different reading thresholds may lead to different reactivation rate estimates.

In conclusion, our study demonstrates the feasibility and effectiveness of LTBI screening and treatment based on long-term outcomes in this cohort. It shows high TB reactivation rates and reaffirms the better predictive value of QFT based screening compared with TST. Based on this long-term initiative there are important lessons about effective prevention of TB reactivation to be considered for the national LTBI screening programme in England and elsewhere, particularly in the context of utilising LTBI screening and treatment in the context of TB elimination targets in low-incidence countries $[3,4]$.

\section{Acknowledgements}

The authors would like to acknowledge the support of the National Institute for Health Research Health Protection Research Unit in Respiratory Infections at Imperial College London (London, UK).

\section{References}

1 World Health Organization. Global Tuberculosis Report 2016. www.who.int/tb/publications/global_report/en/ Date last accessed: February 11, 2017.

2 World Health Organization. Global Strategy and Targets for Tuberculosis Prevention, Care and Control after 2015. www.who.int/tb/post2015_strategy/en/ Date last accessed: May 21, 2017. Date last updated: 2014.

3 Lönnroth K, Migliori GB, Abubakar I, et al. Towards tuberculosis elimination: an action framework for low-incidence countries. Eur Respir J 2015; 45: 928-952.

4 D'Ambrosio L, Dara M, Tadolini M, et al. Tuberculosis elimination: theory and practice in Europe. Eur Respir J 2014; 43: 1410-1420.

5 Getahun H, Matteelli A, Abubakar I, et al. Management of latent Mycobacterium tuberculosis infection: WHO guidelines for low tuberculosis burden countries. Eur Respir J 2015; 46: 1563-1576.

6 Smieja MJ, Marchetti CA, Cook DJ, et al. Isoniazid for preventing tuberculosis in non-HIV infected persons. Cochrane Database Syst Rev 2000; 2: CD001363.

7 Stagg HR, Zenner D, Harris RJ, et al. Treatment of latent tuberculosis infection: a network meta-analysis. Ann Intern Med 2014; 161: 419-428.

8 National Institute for Health and Care Excellence. Tuberculosis. Nice Guideline NG33. www.nice.org.uk/guidance/ ng33 Date last accessed: May 21, 2017. Date last updated: May 2016.

9 World Health Organization. Guidelines on the Management of Latent Tuberculosis Infection. www.who.int/tb/ publications/ltbi_document_page/en/ Date last accessed: May 21, 2017. Date last updated: 2014.

10 Public Health England. Tuberculosis in England 2016 Report. www.gov.uk/government/uploads/system/uploads/ attachment_data/file/581238/TB_Annual_Report_2016_GTW2309_errata_v1.2.pdf Date last accessed: May 21, 2017.

11 Public Health England. Collaborative Tuberculosis Strategy for England: 2015 to 2020. www.gov.uk/government/ publications/collaborative-tuberculosis-strategy-for-england Date last accessed: May 21, 2017. Date last updated: 2015.

12 Control and prevention of tuberculosis in the United Kingdom: Code of Practice 1994. Joint Tuberculosis Committee of the British Thoracic Society. Thorax 1994; 49: 1193-1200.

13 Choudhury IW, West CR, Ormerod LP. The outcome of a cohort of tuberculin-positive predominantly South Asian new entrants aged 16-34 to the UK: Blackburn 1989-2001. J Public Health 2014; 36: 390-395.

14 Newcombe HB. Handbook of Record Linkage. Oxford, Oxford University Press, 1988.

15 Potz N, Powell D. Manual for the Probabilistic Linkage of Infection and Death Records. London, Health Protection Agency, 2007.

16 Aldridge RW, Shaji K, Hayward AC, et al. Accuracy of probabilistic linkage using the enhanced matching system for public health and epidemiological studies. PLoS One 2015; 10: e0136179.

17 Home Office. Migration Statistics. www.gov.uk/government/collections/migration-statistics Date last accessed: December 8, 2016. Date last updated: February 23, 2017.

18 Rubin DB. Multiple Imputation for Nonresponse in Surveys. Hoboken, John Wiley \& Sons, 1987.

19 Diel R, Loddenkemper R, Nienhaus A. Predictive value of interferon- $\gamma$ release assays and tuberculin skin testing for progression from latent TB infection to disease state: a meta-analysis. Chest 2012; 142: 63-75.

20 Rangaka MX, Wilkinson KA, Glynn JR, et al. Predictive value of interferon- $\gamma$ release assays for incident active tuberculosis: a systematic review and meta-analysis. Lancet Infect Dis 2012; 12: 45-55.

21 Diel R, Goletti D, Ferrara G, et al. Interferon- $\gamma$ release assays for the diagnosis of latent Mycobacterium tuberculosis infection: a systematic review and meta-analysis. Eur Respir J 2011; 37: 88-99. 
22 Zellweger J-P, Sotgiu G, Block M, et al. Risk assessment of tuberculosis in contacts by IFN- $\gamma$ release assays. A Tuberculosis Network European Trials Group study. Am J Respir Crit Care Med 2015; 191: 1176-1184.

23 Kruijshaar ME, Abubakar I, Stagg HR, et al. Migration and tuberculosis in the UK: targeting screening for latent infection to those at greatest risk of disease. Thorax 2013; 68: 1172-1174.

24 Pareek M, Watson JP, Ormerod LP, et al. Screening of immigrants in the UK for imported latent tuberculosis: a multicentre cohort study and cost-effectiveness analysis. Lancet Infect Dis 2011; 11: 435-444.

25 Interrante JD, Haddad MB, Kim L, et al. exogenous reinfection as a cause of late recurrent tuberculosis in the United States. Ann Am Thorac Soc 2015; 12: 1619-1626. 